\title{
Territorialização com uso de georreferenciamento e estratificação de vulnerabilidade social familiar na Atenção Básica
}

\author{
Territorialization using georreferencing and stratification \\ of the social vulnerability of families in Primary Care
}

\author{
Monyelle de Oliveira Calistro (https://orcid.org/0000-0001-8343-5752) ${ }^{1}$ \\ Ygor Teixeira (https://orcid.org/0000-0001-6886-9392) ${ }^{2}$ \\ Izabel Ricaelle Argentino Silva Lacerda (https://orcid.org/0000-0003-4719-7480) ${ }^{3}$ \\ Samara Mendes de Sousa (https://orcid.org/0000-0002-5429-3844) ${ }^{1}$ \\ João Agostinho Neto (http://orcid.org/0000-0002-0164-8269) ${ }^{4}$ \\ Sandra Mara Pimentel Duavy (http://orcid.org/0000-0002-1786-2294) ${ }^{1}$ \\ Francisco Elizaudo de Brito Júnior (https://orcid.org/0000-0001-8343-2244) ${ }^{1}$
}

\footnotetext{
${ }^{1}$ Universidade Regional do Cariri. R. Cel. Antônio Luíz 1161, Pimenta. 63105-010 Crato CE Brasil. monna1-oliveira@ hotmail.com

${ }^{2}$ Instituto Federal de Educação, Ciência e Tecnologia do Ceará. Fortaleza CE Brasil. ${ }^{3}$ Universidade Estadual da Paraíba. Campina Grande PB Brasil.

${ }^{4}$ Universidade Federal do Ceará. Fortaleza CE Brasil.
}

\begin{abstract}
This article describes a process of territorialization undertaken in Family Health Strategy micro-areas by a team of residents from the Interprofessional Public Health Residency Program at Cariri Regional University using georeferencing tools and the stratification of families according to degree of social vulnerability. A map of social vulnerability was created using SW Maps and Google Earth Pro based on sociodemographic and clinical data obtained from forms $A$ and $B$ of the e-SUS and inputted into an Excel worksheet. The families were stratified into five degrees of vulnerability based on the overall score obtained for a set of socioeconomic and clinical sentinel indicators: without risk, low risk, medium risk, high risk and very high risk. During the territorialization process, we identified streets and side streets and georeferenced points of risk, social facilities, registered and unregistered families, and vacant homes. Over half of the 615 georeferenced families (316 or $51.38 \%$ ) were not registered in the e-SUS or had not completed their registration at the time of data collection. Most of the 299 registered families (60.53\%) were classified as being at low risk and a considerable portion were medium risk. Key words Geographic mapping, Primary Health Care, Social problems
\end{abstract}

Resumo O presente artigo objetivou descrever a territorialização com uso do georreferenciamento e da construção de mapeamento geográfico e a estratificação de vulnerabilidade social familiar na Atenção Primária à Saúde vivenciado por uma equipe de residentes do programa de Residência Multiprofissional em Saúde Coletiva baseada nos principais problemas sociais. Para territorialização utilizou-se dos programas SW Maps e Google Earth Pro e para caracterização sociodemográfica e clinica das famílias fichas A e B do e-SUS, transcritas em planilha para cálculo da estratificação. Através do score final gerado com o preenchimento de sentinelas, o programa estratificou as famílias em graus de vulnerabilidade de diferentes possibilidades, sendo elas, sem risco, baixo risco, médio risco, alto risco e altíssimo risco. Na territorialização identificou-se ruas, travessas e georreferenciou-se pontos de riscos, equipamentos sociais, famílias com e sem cadastros do e-SUS e casas desocupadas. Das 615 famílias georreferenciadas, $316(51,38 \%)$ não tinham cadastro ou esses estavam incompletos no momento da coleta, enquanto que 299 famílias possuíam cadastro preenchido nas quais observou-se que a maioria (60,53\%) apresentou situação de baixo risco e uma parcela considerável foi considerada de médio risco.

Palavras-chave Mapeamento geográfico, Atenção Primária à Saúde, Problemas sociais 


\section{Introdução}

Considerada porta de entrada preferencial do usuário no Sistema Único de Saúde (SUS) ${ }^{1}$, ordenadora da Rede de Atenção à Saúde (RAS) e o primeiro nível de atenção dentro dessa Rede $^{2}$, a Atenção Básica (AB), também chamada de Atenção Primária à Saúde (APS) compõe um conjunto de ações de saúde desenvolvidas por uma equipe multiprofissional à população de um território e visa impactar positivamente nos determinantes e condicionantes de saúde da coletividade ${ }^{1}$.

A inserção da equipe multiprofissional que compõe a Estratégia Saúde da Família (ESF) no território garante a integralidade nas ações em saúde, a partir do diagnóstico e planejamento situacional estabelecendo maior vínculo e cuidado em saúde, e constitui-se como um dos principais instrumentos de intervenção desta estratégia para o desempenho das ações de vigilância em saúde ${ }^{3,4}$, assim, o processo de territorialização possibilita conhecer as singularidades da vida social e as necessidades em saúde da população ${ }^{5}$ através do cadastramento familiar e da geografia da área, organizando as ações de promoção e prevenção em saúde ${ }^{6}$.

Com o intuito de melhorar a análise e avaliação dos dados em saúde pública, os profissionais podem aliar a territorialização ao Sistema de Informação Geográfica (SIG) ${ }^{7}$, o qual permite processar dados georreferenciados com grande poder de integração e processamento ${ }^{8}$. Esse instrumental tecnológico vem sendo considerado pela Organização Pan-Americana da Saúde (OPAS) como uma das mais efetivas tecnologias existentes para facilitar os processos de decisão e tomadas de decisão em saúde pública9 .

$A$ informatização da $\mathrm{AB}$ tem se confirmado em vários países como uma estratégia importante para automatizar os processos e qualificar a gestão da informação ${ }^{10}$. O Ministério da Saúde (MS) em 2013 com a intenção de reestruturar o Sistema de Informação da Atenção Básica (SIAB) em nível nacional produziu sistemas de software (SUS eletrônico), sendo um deles o de Coleta de Dados Simplificada (CDS $)^{11}$. As fichas do e-SUS $\mathrm{AB}$, incluindo as fichas de cadastro individual e as fichas de cadastro domiciliar são fichas de papel que compõem o CDS e servem para alimentar esse sistema informatizado ${ }^{12}$.

Através da análise das fichas do e-SUS pode-se determinar o risco social e de saúde das famílias adscritas, refletindo o potencial de adoecimento de cada núcleo familiar pela definição de sentinelas e score de risco, como ocorre pela utilização da Escala de Risco Familiar de Coelho e Savassi ${ }^{13}$, estabelecendo assim, de maneira simples, clara e fácil a priorização das visitas domiciliares de acordo com o grau de risco familiar e em microáreas de maior necessidade, sem deixar de cobrir todas as famílias ${ }^{14}$.

O modelo de estratificação de risco desenvolvido pelo programa de Residência Multiprofissional em Saúde Coletiva (RMSC) da Universidade Regional do Cariri (URCA) no projeto de modelagem ecossistêmica articula os processos de ensino, serviço e comunidade, tratando-se de um modelo de vigilância desenvolvido por residentes atuantes em Unidades Básicas de Saúde (UBSs) da cidade do Crato que se utiliza de determinantes sociais e ambientais obtidos pelo processo de territorialização, estratificação de risco familiar ${ }^{15}$.

Com esse perfil epidemiológico são construídos mapas epidemiológicos com a distribuição das famílias de acordo com riscos encontrados utilizando-se de ferramentas tecnológicas são implantadas salas de situação em saúde nas UBSs e construídos matrizes ecossistêmicas de indicadores e planejamento para ações em saúde ${ }^{15}$. Assim, o objetivo deste trabalho é descrever o processo de territorialização com uso do georreferenciamento e estratificação de vulnerabilidade social familiar na atenção básica vivenciado por uma equipe de residentes do programa de RMSC.

\section{Método}

Trata-se de um estudo descritivo com abordagem quantitativa a partir da aplicação das tecnologias de georreferenciamento e estratificação de vulnerabilidade social familiar, baseada nos principais problemas sociais, durante um processo de territorialização realizado no período de março de 2019 a março de 2020 pela turma 3 do programa de RMSC da URCA.

Foram realizadas incursões no território pela turma da RMSC acompanhados pelos Agentes Comunitários de Saúde (ACS), responsáveis pelas microáreas. A territorialização foi realizada na ESF do Parque Grangeiro II, Crato, Ceará. A equipe da ESF é composta por um médico, uma enfermeira, uma técnica em enfermagem, quatro ACS, um auxiliar administrativo e uma auxiliar de serviços gerais. As geotecnologias foram utilizadas para um melhor conhecimento da área e classificação das famílias adscritas do território em graus de vulnerabilidade socioeconômicos e clínicos. 
O estudo foi desenvolvido em duas etapas. A primeira ocorreu entre abril a setembro de 2019 , com a territorialização das quatro microáreas: Microárea I: Vila Gregório e Vila Pedrosa, Microárea II: Vila Nova, Microárea III: Sítio Coqueiro e Microárea IV: Sítio Caiana.

Utilizou-se um aplicativo de celular gratuito disponível para Android, o SW Maps que possibilita carregar shapefiles, simbolizar com base nos atributos, navegação, coleta de dados via GPS e manualmente, além de exportar e compartilhar informações via Android ${ }^{16}$. Através da latitude e longitude, pode-se georreferenciar os locais do território com adição de pontos que ao exportá-los para um outro programa, o Google Earth Pro, foi gerado um mapeamento geográfico do território georreferenciado. O Google Earth Pro trata-se de uma tecnologia avançada que permite uma perspectiva tridimensional de qualquer local do mundo ${ }^{17}$.

$\mathrm{Na}$ segunda etapa foi realizada a caracterização sociodemográfica e clínica das famílias adscritas através das fichas do e-SUS de cadastro familiar e individual e posteriormente transcritas em planilha do Microsoft Excel elaborada para cálculo da estratificação da vulnerabilidade familiar. Através do score final gerado com o preenchimento de sentinelas ilustradas no Quadro 1 o programa estratificou as famílias em graus de vulnerabilidade de cinco diferentes possibilidades, sendo elas, sem risco, baixo risco, médio risco, alto risco e altíssimo risco (Quadro 2).

A pesquisa foi aprovada pelo Comitê de Ética em pesquisa da URCA.

\section{Resultados}

A equipe da ESF Parque Grangeiro II atua em quatro microáreas, e cada uma tem como responsável um ACS, sendo elas Microárea I: Vila Gregório e Vila Pedrosa, Microárea II: Vila Nova, Microárea III: Sítio Coqueiro e Microárea IV: Sítio Caiana. As duas últimas são localizadas na zona rural, no entanto o Sítio Coqueiro é totalmente da zona rural. As microáreas mais distantes da UBS são a III e a IV.

$\mathrm{Na}$ territorialização, através da ferramenta do SW Maps identificou-se as ruas, travessas e georreferenciou-se pontos de riscos, equipamentos sociais, famílias com e sem cadastros do e-SUS e casas desocupadas, obtendo como resultado o Quadro 3.

Das 615 famílias georreferenciadas, 316 $(51,38 \%)$ não tinham cadastro familiar no e-SUS ou esses estavam incompletos no momento da coleta, mesmo nas microáreas mais próximas da UBS. As famílias do território foram classificadas em cinco graus de vulnerabilidade de acordo com suas condições socioeconômicas e clínicas. A Figura 1 consiste numa imagem retirada do Google Earth Pro da vulnerabilidade familiar das quatro microáreas.

Do total de 299 famílias com cadastro preenchido das fichas A e B do e-SUS observou-se que a maioria $(60,53 \%)$ apresentou situação de baixo risco e uma parcela considerável foi considerada de médio risco (Quadro 4). Das variáveis das sentinelas de condições socioeconômicas presentes no Quadro 1, as que mais contribuíram para situação de risco das famílias foram: fossa rudimentar como forma de escoamento do banheiro de $44,14 \%$ das famílias, seguido de chão batido como tipo de acesso ao domicílio (42,47\%) e água clorada como água para consumo $(34,44 \%)$. Outro dado socioeconômico relevante foi a variação na renda per capita mensal das famílias entre as microáreas, na I e na II, respectivamente $49,10 \%$ e $45,67 \%$ das famílias possuíam renda per capita mensal de até RS 238,00 enquanto que para a microárea III esse valor correspondeu apenas a $10,93 \%$ das famílias, e na microárea IV a 23,80\% em que valores de renda per capita superiores foram encontrados para maioria das famílias.

As variáveis das sentinelas das condições clínicas que mais pontuaram foram usuário de álcool (14,97\%), Hipertensão Arterial Sistêmica (13,90\%) e Diabetes mellitus (4,38\%) nos 935 usuários cadastrados.

\section{Discussão}

Considerável número de pontos de risco foram localizados durante o processo de territorialização, locais propícios a situações de maior vulnerabilidade para comunidade, como esgoto a céu aberto, terrenos baldios, casas abandonadas, que configuram problemas de segurança e saúde pública por servirem como depósito de entulho e lixo para moradores circunvizinhos podendo resultar em acúmulo de água parada e possíveis criadouros para mosquitos como Aedes aegypti, transmissor de arboviroses (dengue, zika, chikungunya e febre amarela). Esses terrenos também podem servir de esconderijo para marginais e delinquentes após prática de violência ou assalto $^{18}$.

Ao longo do território foram georreferenciados pontos poluídos do rio Granjeiro, principal 
Quadro 1. Pontuação de cada variável socioeconômica e clínica das sentinelas e condições para pontuar pela renda per capita.

\begin{tabular}{|c|c|c|}
\hline Sentinelas condições socioeconômicas & Variável & Pontuação \\
\hline Situação de Moradia & Situação de Rua & 2 \\
\hline Tipo de Acesso ao Domicílio & Chão Batido/ Fluvial & 1 \\
\hline Disponibilidade de Energia Elétrica & Não & 1 \\
\hline Relação No Cômodos/No Moradores & $<1$ & 1 \\
\hline Relação No Cômodos/No Moradores & $<0.3$ & 2 \\
\hline Material Predominante na Construção da Casa & $\begin{array}{l}\text { Alvenaria sem Revestimento/ Taipa/ } \\
\text { Material Aproveitado/Palha }\end{array}$ & 1 \\
\hline Água para Consumo no Domicílio & Clorada/ Sem tratamento & 1 \\
\hline Forma de Escoamento do Banheiro ou Sanitário & $\begin{array}{l}\text { Fossa Rudimentar/ Direto para um Rio, } \\
\text { Lago ou Mar/ Céu aberto }\end{array}$ & 1 \\
\hline Destino do Lixo & Queimado/Enterrado/ Céu Aberto & 1 \\
\hline Presença de cuidador para idoso Analfabeto & Sim & 1 \\
\hline Presença de crianças sozinhas ou sob cuidado de outra & Sim & 1 \\
\hline Sentinelas condições clínicas & No de casos & Pontuação \\
\hline $\begin{array}{l}\text { Criança(s) de } 0 \text { a } 2 \text { Anos/ Gestante(s)/ Usuário(s) de } \\
\text { Álcool/ Hipertenso(s)/Diabético(s)/ Portador(es) de } \\
\text { Riscos Cardiovasculares/ Paciente(s) com Disfunção } \\
\text { Renal/ Portador(es) de Doenças Respiratórias/ } \\
\text { Portador(es) de Hanseníase/ Portador(es) de } \\
\text { Tuberculose/ Idoso(s) Acima de } 80 \text { Anos }\end{array}$ & 1 & 1 \\
\hline $\begin{array}{l}\text { Indivíduo(s) com Deficiência/ Usuário(s) de Outras } \\
\text { Drogas/ Portador(es) de Câncer /Portador(es) } \\
\text { Problemas de Saúde Mental/ Paciente(s) Acamado(s) }\end{array}$ & 1 & 2 \\
\hline Renda Familiar Mensal & No de Membros no domicílio & Pontuação \\
\hline 1/4 de salário & 2 & 1 \\
\hline $1 / 2$ salário & 3 & 1 \\
\hline 1 salário & 5 & 1 \\
\hline 2 salários & 9 & 1 \\
\hline 3 salários & 13 & 1 \\
\hline 4 salários & 17 & 1 \\
\hline
\end{tabular}

Fonte: Programa de RMSC - URCA.

Quadro 2. Graus de risco familiar de acordo com o score final.

\begin{tabular}{|l|c|}
\hline \multicolumn{1}{|c|}{ Graus de risco } & Score \\
\hline Sem Risco & 0 \\
\hline Baixo Risco & $1-3$ \\
\hline Médio Risco & $4-6$ \\
\hline Alto Risco & $7-8$ \\
\hline Altíssimo Risco & $9-10$ \\
\hline
\end{tabular}

Fonte: Programa de RMSC - URCA.

rio urbano municipal cuja bacia inicia-se na Chapada do Araripe e termina no Rio Batateiras $^{19}$. Identificou-se presença de matérias inorgânicas e entulhos ao longo do rio, enchentes (principalmente em tempos de chuvas), e moradores que lançam seus desejos sanitários diretamente no rio por não possuírem forma de escoamento adequada. A poluição dos ecossistemas aquáticos, o desmatamento, a urbanização mal planejada, com ocupação indevida de áreas próximas dos cursos d’águas, transformam drasticamente a natureza desses cursos locais o que traz impacto sobre o meio ambiente e os habitantes ${ }^{20}$.

Outro dado importante identificado no processo de territorialização foi a escassez de equipamentos sociais, o que demanda uma atenção por parte dos gestores com o plano diretor do município para ampliação e/ou criação dos que ainda não existem que são escolas, creches, farmácias, clínicas odontológicas, academias de ginástica, parques, laboratórios, telefones públicos, entre outros. 
Quadro 3. Georreferenciamento de alguns pontos do território pertencente à ESF Parque Grangeiro II. CratoCE, Brasil, 2019.

\begin{tabular}{|l|c|c|c|c|c|}
\hline \multicolumn{1}{|c|}{ Pontos georreferenciados } & $\begin{array}{c}\text { Vila Gregório/ } \\
\text { Vila Pedrosa }\end{array}$ & Vila Nova & $\begin{array}{c}\text { Sítio } \\
\text { Coqueiro }\end{array}$ & Sítio Caiana & Total \\
\hline Ruas e travessas & 17 & 14 & $\star$ & $\star$ & $*$ \\
\hline Pontos de risco & 66 & 21 & 21 & 55 & 163 \\
\hline Equipamentos sociais & 05 & 03 & 06 & 04 & 18 \\
\hline Famílias com cadastro do e-SUS & 112 & 81 & 42 & 64 & 299 \\
\hline Famílias sem Cadastro do e-SUS & 149 & 66 & 58 & 43 & 316 \\
\hline $\begin{array}{l}\text { Total de indivíduos com cadastro } \\
\text { concluído no e-SUS }\end{array}$ & 362 & 253 & 139 & 181 & 935 \\
\hline Casas desocupadas & 06 & 08 & 14 & 21 & 49 \\
\hline
\end{tabular}

*Por se tratarem de microáreas de zona rural, não existe nomes de ruas nem de travessas em determinados lugares.

Fonte: Elaborado pelos autores.

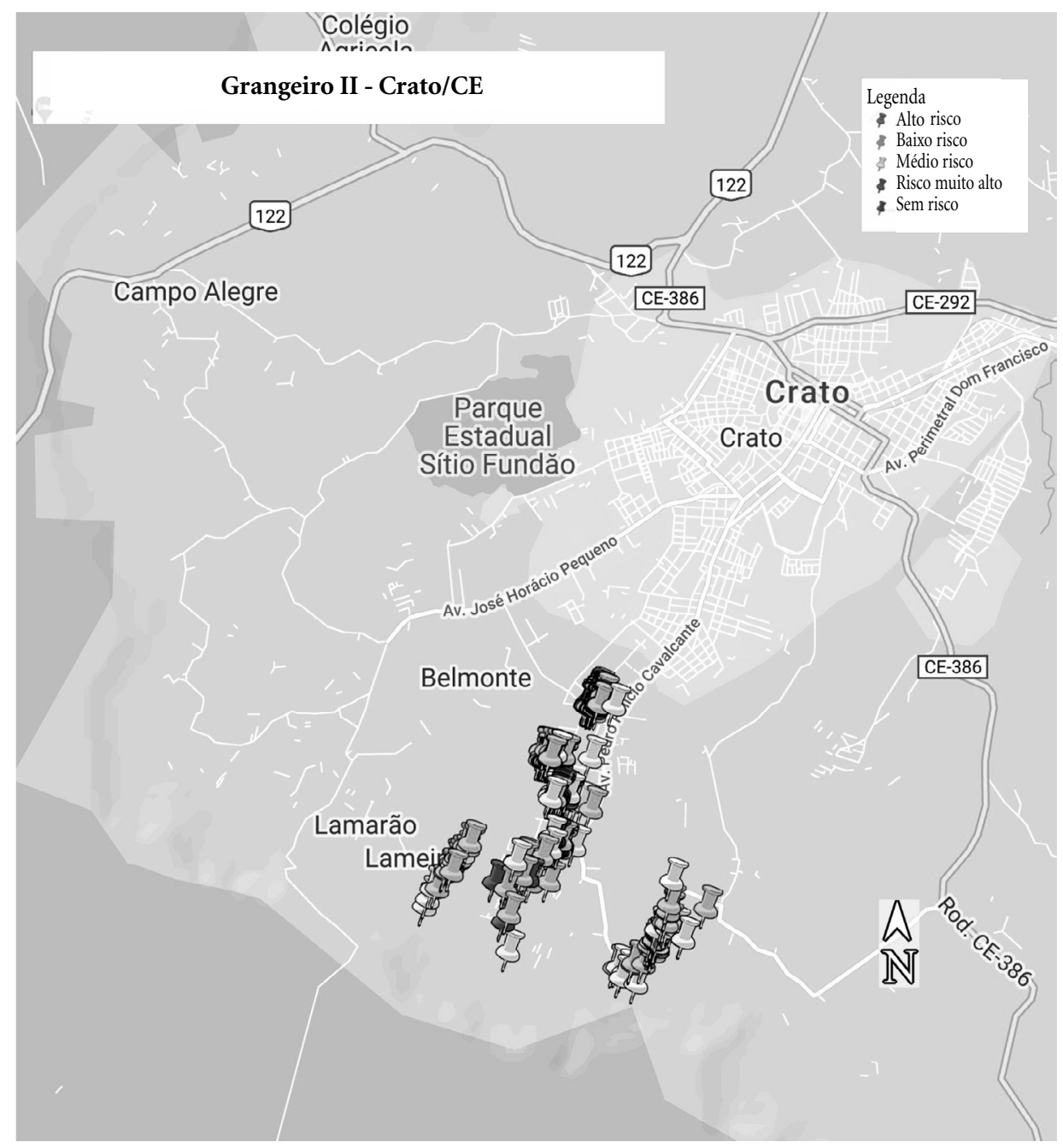

Figura 1. Vulnerabilidade social das famílias cadastradas no e-SUS do território pertencente a ESF do Parque Grangeiro II. Crato-CE, Brasil, 2019. 
Quadro 4. Vulnerabilidade social das famílias do território pertencente à ESF Parque Grangeiro II por microárea. Crato-CE, Brasil, 2019.

\begin{tabular}{|l|c|c|c|c|c|c|}
\hline Vulnerabilidade familiar & $\begin{array}{c}\text { Vila Gregório/ } \\
\text { Vila Pedrosa }\end{array}$ & Vila Nova & $\begin{array}{c}\text { Sítio } \\
\text { Coqueiro }\end{array}$ & Sítio Caiana & Total & \% Total \\
\hline Famílias sem risco & 0 & 0 & 0 & 9 & 9 & 3,01 \\
\hline Famílias com baixo risco & 67 & 55 & 27 & 32 & 181 & 60,53 \\
\hline Famílias com médio risco & 38 & 25 & 11 & 22 & 96 & 32,10 \\
\hline Famílias com alto risco & 06 & 1 & 3 & 1 & 11 & 3,67 \\
\hline Famílias com altíssimo risco & 1 & 0 & 1 & 0 & 2 & 0,66 \\
\hline Total de famílias & 112 & 81 & 42 & 64 & 299 & \\
\hline
\end{tabular}

Fonte: Elaborado pelos autores.

Atualmente, estudos como o de Neves apontam para o fato de que a responsabilidade para estipular critérios relacionados ao planejamento de equipamentos urbanos comunitários caber diretamente aos planos diretores municipais, possibilita a implantação desses de forma pouco sustentável, pouco acessível à população, mal distribuídos e mal dimensionados, baseada na realidade de outros municípios e não nas necessidades locais ${ }^{21}$.

Consideram-se comunitários os equipamentos públicos de educação, cultura, saúde, lazer e similares $^{22}$, vale destacar que no território não existe nenhuma escola ativada, apenas o espaço físico de uma unidade escolar no Sítio Coqueiro desativada, o que faz com que os alunos necessitem se deslocar para escolas fora do espaço territorial, que é um fator desestimulante ao ingresso e permanência desses no ensino escolar ${ }^{23}$. A acessibilidade de equipamentos públicos está relacionada às características destes ambientes, porque os comportamentos são influenciados pelo ambiente que os cercam, que facilita, inibe ou define ações ${ }^{24}$.

No território, os locais de prática de exercícios físicos ficam restritos aos poucos campos de futebol, campos esportivos privados e a associação dos moradores presente na microárea Vila Nova, na qual atualmente acontece um projeto inaugurado pela residente em educação física voltado às mulheres das microáreas próximas com práticas de exercícios físicos, relaxamento muscular e rodas de conversas orientando para o cuidado integral em saúde. A escassez de equipamentos sociais com espaço para prática de exercícios físicos, como campos de futebol, academias de ginástica, clubes recreativos reflete diretamente na saúde da comunidade propiciando o desenvolvimento de doenças crônica.

A Política Nacional da Atenção Básica (PNAB) de 2017 define como atribuição comum a todos profissionais que atuam na $\mathrm{AB}$ cadastrar e manter atualizado o cadastramento e outros dados de saúde das famílias e dos indivíduos no sistema de informação da $\mathrm{AB}$ vigente, no entanto destaca como de responsabilidade do ACS cadastrar todas as pessoas de sua área, mantendo os dados atualizados por meio das visitas domiciliares $^{1}$, Na estratificação da vulnerabilidade familiar, apesar da importância dos cadastros familiares para o diagnóstico situacional em saúde menos da metade das famílias georreferenciadas tinham o cadastro do e-SUS completo no momento da análise, fato que dificultou o levantamento do estudo.

Vários são os motivos que podem ter contribuídos para o não preenchimento das fichas, um dos quais consiste no fato de nesse território existir um número considerável de famílias com elevado poder aquisitivo que possuem plano de saúde privado e não procuraram atendimento no SUS. Um estudo feito por Baralhas e Pereira ${ }^{25}$ mostrou que uma das dificuldades enfrentadas pelo ACS na execução de suas práticas diárias está na falta de tempo de alguns usuários para recebê-los e na postura que alguns adotam em não aceitar suas visitas, por não entenderem os benefícios que o trabalho desses profissionais traz para as ações de prevenção de doenças e promoção da saúde.

Essa desatualização dos cadastros familiares acontece em outras ESFs como no município de Salinas, norte de Minas Gerais, o qual foi descrito como problema prioritário na ESF Floresta $\mathrm{I}^{26}$. A PNAB de 2017 traz ainda uma atualização no número de pessoas que pode ficar sob responsabilidade de cada ACS dentro do território, que deve ser de no máximo 750 pessoas por ACS, independentemente do número de famílias da microárea. Como existe uma quantidade de famílias sem cadastro no território do Parque Grangeiro II, no momento da análise, não teve como confrontar 
os dados para identificar se a população de cada um desses profissionais ultrapassou 750 pessoas.

As situações de baixo risco e médio risco foram as mais prevalentes na estratificação da vulnerabilidade familiar, fato semelhante ao encontrado no Um trabalho feito por Nataka et al. ${ }^{27}$ realizado em uma ESF de Porto Alegre em 2013 através da consulta de dados da ficha A do SIAB mostrou que uma proporção significativa (31,5\%) de 927 famílias avaliadas foi classificada com algum grau de risco, sendo a maioria de menor risco. As variáveis mais frequentes para situação de risco nesse estudo foram baixas condições de saneamento, Hipertensão Arterial Sistêmica e drogatização, respectivamente.

Com relação às condições socioeconômicas apresentadas ao longo do território, a renda per capita mensal das famílias chamou atenção por demonstrar grande variação entre as microáreas. Vale salientar que o bairro Parque Grangeiro II possui famílias de um poder aquisitivo bastante elevado, enquanto as famílias que moram nos arredores da UBS apresentam em sua maioria baixa condição social.

Um estudo feito por Tavares ${ }^{28}$ trouxe algumas razões que contribuem para que famílias com alto poder aquisitivo sejam atraídas para residir no território do Parque Grangeiro, uma possível causa está na tendência de pessoas com alto poder aquisitivo procurar por locais distantes das áreas de maior aglomeração de pessoas, pela localização no sopé da Chapada do Araripe e possuir uma considerável área de proteção ambiental cuja condição atmosférica local apresenta um clima mais ameno do que cidades vizinhas.

\section{Considerações finais}

O processo de territorialização permitiu o diagnóstico situacional de toda área territorial pertencente à ESF do Parque Grangeiro II, identificando suas diversidades, vulnerabilidades, fragilidades, potencialidades, sendo muito relevante, tendo em vista que utilizou-se de ferramentas avançadas como estratégias inovadoras para equipe do território possibilitada pela inserção da Residência Multiprofissional em Saúde da Família da URCA na ESF. A aplicação dessas geotecnologias mostrou-se eficaz uma vez que facilitou a geração, o armazenamento, a localização bem como a análise de dados de saúde da população.

A estratificação de vulnerabilidade familiar no território do Parque Grangeiro II, por meio de scores de riscos, outra prática inovadora para unidade teve como principal limitação o fato de boa parte das famílias georreferenciadas não possuírem cadastro familiar no e-SUS ou esses estavam incompletos no momento da análise, apesar da importância dos cadastros familiares para o diagnóstico situacional em saúde. Mais da metade das famílias cadastradas foram classificadas como de baixo risco e uma parcela considerável de famílias de médio risco resultado semelhante ao de outros estudos encontrados na literatura. Quanto à presença das sentinelas de risco das condições clínicas, as referentes à usuário de álcool, Hipertensão Arterial Sistêmica e Diabetes mellitus foram as que mais pontuaram nos 935 usuários cadastrados. Esse levantamento tem como principal vantagem facilitar o planejamento estratégico, para que o mesmo ocorra de forma equitativa, priorizando as famílias com maiores riscos.

A relevância dessa experiência está ainda no despertar da consciência dos residentes para importância de conhecer o território e estabelecer nele um olhar vigilante para que as ações em saúde desenvolvidas pela equipe aconteçam privilegiando aspectos essenciais à vida das pessoas que habitam nele, enxergando-o como espaço vivo, que está em constante transformação e a utilização de sistemas de informações trouxe agilidade no processo e maior visibilidade dos resultados encontrados, mostrando-se muito útil no que se propôs, devendo pois ser estimulada nos processos de trabalho das ESFs.

\section{Colaboradores}

MO Calistro: trabalhou na escolha do tema, na coleta de dados, análise e interpretação, na pesquisa e metodologia, na redação do artigo, na concepção e elaboração do texto final, além de trabalhar na adequação às normas para a revista. SM Souza e IRAS Lacerda: coleta e análise de dados. Y Texeira: formatação geral do trabalho, incluindo edição de referências, texto geral, produção de imagem e edição das tabelas apresentadas, auxiliando também na seleção das palavraschave e no processo de submissão no endereço eletrônico indicado pela revista. FE Brito Júnior, SP Duavy: atuaram na metodologia, administração de projetos, supervisão, revisão de redação e edição do artigo. J Agostinho Neto: atuou na metodologia e revisão de redação e edição do artigo. 


\section{Referências}

1. Brasil. Portaria no 2.436, de 21 de setembro de 2017. Aprova a Política Nacional de Atenção Básica, estabelecendo a revisão de diretrizes para a organização da Atenção Básica, no âmbito do Sistema Único de Saúde (SUS). Diário Oficial da União; 2017.

2. Brasil. Decreto no 7.508, de 28 de junho de 2011. Regulamenta a Lei no 8.080 , de 19 de setembro de 1990 , para dispor sobre a organização do Sistema Único de Saúde - SUS, o planejamento da saúde, a assistência à saúde e a articulação interfederativa, e dá outras providências. Diário Oficial da União 2011; 19 set.

3. Silva Júnior ESD, Medina MG, Aquino R, Fonseca ACF, Vilasbôas ALQ. Acessibilidade geográfica à atenção primária à saúde em distrito sanitário do município de Salvador, Bahia. Rev Bras Saude Mater Infant 2010; 10(Supl. 1):s49-s60.

4. Carneiro Junior N, Jesus CHD, Crevelim MA. A estratégia saúde da família para a equidade de acesso dirigida à população em situação de rua em grandes centros urbanos. Saude Soc 2010; 19(3):709-716.

5. Monken M, Barcellos C. O território na promoção e vigilância em saúde. Rio de Janeiro: EPSJV; 2007.

6. Silva DM. Atualização do cadastramento das famílias na Estratégia Saúde da Família: elaboração de um plano de intervenção. [monografia]. Montes Claros: Universidade Federal de Minas Gerais; 2018.

7. Câmara G, Davis C, Monteiro AMV. Introdução à ciência da geoinformação. São José dos Campos: INPE; 2001.

8. León MEDS. SIG na Saúde Pública - Estudo de caso: mortalidade infantil em Dom Pedrito/RS [dissertação]. Santa Catarina: Universidade Federal de Santa Maria; 2007.

9. Organização Panamericana de Saúde (OPAS). Sistemas de informação geográfica em saúde: conceitos básicos. Brasília: OPAS; 2002.

10. Montague $\mathrm{E}$. The promises and challenges of health information technology in primary health care. Primary Health Care. Prim Health Care Res Dev 2014; 15(3):227-230

11. Brasil. Ministério da Saúde (MS). Secretaria de Atenção à Saúde. Departamento de Atenção Básica. Diretrizes Nacionais de Implantação da Estratégia e-SUS Atenção básica. Brasília: MS; 2014.

12. e-SUS Atenção Básica Manual Online [Internet]. [acessado 2020 jun 20]. Disponível em: http://www2. eerp.usp.br/Nepien/ManualeSUS/CDS.html.

13. Coelho FLG, Savassi, LCM. Aplicação de Escala de Risco Familiar como instrumento de priorização das Visitas Domiciliares. Rev Bras Med Fam Comunidade 2004; 1(2):19-26.

14. Costa ACI, Araújo DD, Melo JAS, Rafael MEPPB. Aplicabilidade e limitações da Escala de risco familiar de Coelho e Savassi para o processo de trabalho da enfermagem na atenção básica em saúde. Relato de experiência da Unidade de Saúde da Família de Saúde da Família Macaxeira/Buriti. In: $2^{\circ}$ Seminário Nacional de Diretrizes para Enfermagem na Atenção Básica a Saúde. Recife: ABEN; 2009. p.98-100.
15. Rodrigues LM, Amorim ARB, Moura BRD, Mota DDN, Júnior FEDB. Modelagem Ecossistêmica para Vigilância em Saúde na Atenção Básica. In: XVII Congresso do COSEMS/CE. Rev Sustentação 43. Crato; 2018.

16. SWMaps - Candidato a substituto do ArcPAD? [Internet]. [acessado 2020 maio 26]. Disponível em: http://forest-gis.com/2017/08/swmaps-candidato-asubstituto-do-arcpad.html/.

17. Siqueira TA, Deus SPD. Google Earth Pro: Possibilidades para o estudo da cidade no ensino de geografia. In: Anais do IX Fórum Nacional NEPEG de formação de professores de geografia.

18. Tavares GG, Santos OP, Rosseto LP, Bernardes GD. Território e riscos ambientais: Perfil da área de abrangência da ESF-bairro de Lourdes. Hygeia 2016; 13(23):81-99.

19. Moreira AAC. Modelagem hidrológica da bacia hidrográfica do rio Granjeiro- Crato-CE: composição do cenário atual e simulação de uso e ocupação do solo [dissertação]. Juazeiro do Norte: Universidade Federal do Ceará; 2013.

20. Botelho MHC. Águas de chuva: engenharia das águas pluviais nas cidades. 4a ed. São Paulo: Blucher; 2017.

21. Neves FH. Planejamento de equipamentos urbanos comunitários de educação: algumas reflexões. Cad Metropole 2015; 17(34):503-516.

22. Brasil. Lei $n^{\circ}$ 6.766, de 19 de dezembro de 1979. Dispõe sobre o Parcelamento do Solo Urbano e dá outras Providências. Diário Oficial da União 1979; 19 dez.

23. Silva ADP, Azevedo SDC. A escola como território: relações de poder e políticas educacionais. Cad Geogr 2019; 29(2):55-69.

24. Rio DV. Introdução ao desenho urbano no processo de planejamento. São Paulo: Pini: 1990.

25. Baralhas M, Pereira MAO. Prática diária dos agentes comunitários de saúde: dificuldades e limitações da assistência. Rev Bras Enferm 2013; 66(3):358-365.

26. Silva DM. Atualização do cadastramento das famílias na Estratégia Saúde da Família: elaboração de um plano de intervenção [monografia]. Montes Claros: Universidade Federal de Minas Gerais; 2018.

27. Nakata PT, Koltermann LI, Vargas KR, Moreira PW, Duarte ÊRM, Rosset-Cruz I. Classificação de risco familiar em uma Unidade de Saúde da Família. Rev Lat-Am Enferm 2013; 21(5):1088-1095.

28. Tavares CAJDS. Desastres ambientais: Análise de caso no bairro Granjeiro, em Crato/CE. Rev Tocantinense Geogr 2019; 8(15):54-63.

Artigo apresentado em 22/10/2020

Aprovado em 16/12/2020

Versão final apresentada em 18/12/2020

Editores-chefes: Maria Cecília de Souza Minayo, Romeu Gomes, Antônio Augusto Moura da Silva 